\title{
Erwiderung zu der Veröffentlichung von E. Brack: „Kasuistisches, Statistisches und Theoretisches über den unerwarteten Tod aus innerer Ursache".
}

\author{
Von
}

Dr. Günther Weyrich, Graz.

In der Dtsçh. Z. gerichtl. Med. 19, 179 bezeichnet es Herr E. Brack in seiner Veröffentlichung: Kasuistisches, Statistisches und Theoretisches über den unerwarteten Tod, ,aus innerer Ursache" als großen Vorteil, daß ,gegenüber Zusammenstellungen aus Sektionsbefunden vieler Gutachter, wie sie Weyrich jüngst in diesen Blättern gegeben hat", bei seinem Ausgangsmaterial sämtliche Sektionen von ihm oder unter seiner Leitung ausgeführt wurden. Hierzu erlaube ich mir zu bemerken, daß sich Herr Brack bezüglich der Begutachtung meines Obduktionsmateriales im Irrtum befindet. Zur Aufklärung dieses Mißverständnisses möchte ich auf folgende Tatsachen hinweisen.

Das meiner Arbeit zugrunde liegende Obduktionsmaterial von 2668 plötzlichen Todesf: Ilen bei Erwachsenen stammt teils aus dem Gerichtlich-medizinischen Institute in Wien aus den Jahren 1914-1917 und teils aus dem Gerichtlich-medizinischen Institute in Graz aus der Zeit von 1919 bis Ende 1929. Die Obduktionen in Wien nahm fast ausschließlich mein Chef, Herr Professor Reuter, selbst vor. Die Todesfälle aus dem Grazer Institut wurden wiederum zum Teil von Herrn Professor Reuter selbst bzw. unter seiner Leitung von seinen Schülern, den Herren Schwarzacher und Laves, sowie von mir obduziert. Es besteht somit die von Herrn Brack hervorgehobene Annahme, daß bezüglich der Einheitlichkeit in der Beurteilung und Durcharbeitung meines Materiales nicht die gleichen Bedingungen zutreffen, wie er sie für die von ihm ausgewerteten Fälle in Anspruch nimmt, nicht zu Recht. Daher ist aus der einheitlichen Begutachtung allein ein Vorteil der Zusammenstellung von rund 600 plötzlichen Todesfällen durch Herrn Brack gegenüber meiner Statistik mit 2668 Fällen nicht zu ersehen.

Im übrigen verweise ich auf die genaueren Ausführungen, die im Band 12 der Beitr. gericht. Med. erscheinen. 